\title{
UBC Domain
}

National Cancer Institute

\section{Source}

National Cancer Institute. UBC Domain. NCI Thesaurus. Code C13907.

The 16-kD UBC domain contains a conserved UBC motif and a conserved cysteine that accepts activated ubiquitin from E1 enzyme to form a thiol ester. Basic residues at the UBC domain N-terminus may be involved in E1 binding. UBC/E2 enzymes catalyze ubiquitination of target proteins with or without E3 ' $\mathrm{N}$-end' recognizing proteins. E2s can be classified on the basis of UBC structure. Poor at transferring ubiquitin to proteins on their own, Class I proteins comprise simply the UBC domain and probably require an E3 to ubiquitinate short-lived abnormal proteins prior to degradation. Class II enzymes contain different acidic C-terminal extensions of the UBC domain that appear to involve cellular localization or to mediate interaction with protein substrates for ubiquitination that results in protein modification but not degradation. Class III E2s have $\mathrm{N}$-terminal extensions, but their function is unknown. $(\mathrm{NCl})$ 